\title{
A Preliminary Note on the Cocoon Fungus ("Uchikabi").
}

\author{
$\mathrm{BY}$
}

H. Nomura.

The Cocoon fungus ("Uchikabi") is a well-known malady, causing annually such serious loss to sericulture in this country, that its prevention and destruction are of great importance to sericulturists, and yet no scientific investigations concerning the fungus beyond that published by $R_{A u x}{ }^{(1)}$ seem to have been made ${ }^{(2)}$

$R_{\text {AUX }}$ states that the cocoon fungus is caused by certain species of Aspergillus, and suggests only as a provisional mode of its prevention that the suffocated cocoon should be kept clean and be preserved in a well ventilated dry place; but he has not ascertained the species of the fungi. It may, therefore, be of some interest to give here a brief note of some investigations concerning the fungus which have been made by me in the Botanical Laboratory of the College of Science, Imperial University, under the direction of Prof. M. Mryoshi, with materials received from the Sericultural Experiment Station and other localities.

In the earlier stage of the affection, the outer surface of the cocoon shows nothing particular; but sericulturists can detect its presence even then by the odour of the cocoon, characteristic of the fungus, and also by dullness of the sound heard when the cocoon is shaken close to the ear, instead of the sharp sound heard on shaking a healthy cocoon.

Later on, the surface of the cocoon becomes at some spot slightly discoloured, and then the discolouration slowly spreads and deepens in shade, until the greater part of the cocoon attains a yellowish brown and often a grey hue. When such a cocoon is cut open, its inner surface and the pupa within are found to be greenish, or yellowish brown, or partly one and the other, owing to the presence of different fungi.

(1) Gabriel Raux, Note sur une maladie des cocons causie par un Aspergillus. Laboratoire d'Etude de la soie. 1891, Lyon.

(2) The only literature concerning the cocoon fungus became known to me through the kindness of Prof, Uerson, Director of R. Stazione Bacologica sperimentale, Padova. 
For the isolation of species, I have used Esmarch tubes or roll cultures and the materials were taken from cocoons in different stages of the affection.

Among the bacteria and fungi which have been isolated in this way, two species of Aspergillus, $A$. glaucus and A. flavus, have been found invariably developed in the culture.

On inoculating a number of unaffected cocoons with spores of these two fungi taken from my pure cultures, I have found, after two weeks, the surface of the cocoons presenting the characteristic yellowish brown hue, owing to the more luxuriant growth of A. flavus than of A. glaucus.

We may, therefore, look upon the cocoon fungus as formed chiefly of these two species.

On examining a great number of affected cocoons, I have found that the inner surface of the cocoon and especially the body of the pupa within are in most cases, affected earlier than the outer surface of the cocoon, and also to a much greater extent.

Thus, the part first affected seems to be the body of the pupa rather than the outer surface of the cocoon.

But whether the spores of the two fungi are infected through the mouth or through the spiracles of the larve is a question.

RAUX (1) States that the disease is especially conspicuous, in its earlier stage, on the spiracles of the pupa, but his statement rests upon macroscopical observation only.

To elucidate the question, I have fed a number of the larve for some hours on mulberry leaves whose edges had been rubbed with spores of the fungi, all known precautions being taken during the feeding to prevent the spores getting attached to any other part of their bodies than their mouths; an equal number of larvæ to whose spiracles spores of the fungi had been applied, were fed, with similar precautions, on mulberry leaves, which had received no spores; and an equal number more of the larve were fed in the normal way to serve as control specimens.

Four or five days after the cocoons had been formed by these larvæ, I dried all the cocoons for eight hours at a temperature of $70^{\circ}-75^{\circ} \mathrm{C}$, that being the temperature at which silk growers dry the cocoons. Each cocoon was left for three weeks in a sterilised closed moist chamber, and then cut open and the pupa examined.

In this way, I found that the pupæ to whose spiracles while in their

(1) Gabriel Ranx. loc. cit. p. 73 . 
larval stage spores had been applied to be very strongly affected by the fungi, especially by $A$. flavus, and also their cocoons.

The dung of the larvæ, which had been fed on the mulberry leaves with the spores and of those, on whose spiracles the spores had been rubbed were kept in a sterilised moist chamber.

After some ten days there was observed a luxuliant growth of the fungi on that of the larvi, fed on mulberry leaves with the spores, and only a very weak development of fungi on that of the larve, whose spiracles had been rubbed with the spores. It is very probable that in the latter case the fungi developed had not come from the spores on the spiracles.

I have also made an experiment to see whether or not the germs of these fungi are commonly floating in the air of the rearing room, and have found them to be there.

Conclusions :-

1) Cocoon fungus ("Uchikabi") is chiefly due to the attack of two parasitic fungi, Aspergillus flavus and A. glaucus, and of the former more than the latter.

2) The germs of these fungi are actually floating in the air of the rearing room.

3) The fungi seem to obtain entrance into the larvæ through the spiracles rather than through the mouth.

4) The cocson fungus thus seems to begin in the body of the pupa and thence extend gradually to the cocoon, where it finally makes its way to the outer surface.

5) The spores of these fungi do not lose their vitality on passing through the alimentary canal of the silkworm.

6) Desiccation for eight hours at the temperature of $70^{\circ}-75^{\prime} \mathrm{C}$., is not efficient for killing the spores of the fungi.

Sericultural Experiment Station,

Nishigahara, Tōkyō, Japan,

May 9th, 1897. 